Algebra univers. 45 (2001) $103-106$

$0002-5240 / 01 / 010103-04 \$ 1.50+0.20 / 0$

(C) Birkhäuser Verlag, Basel, 2001

Algebra Universalis

\title{
Mailbox
}

\section{The dualisability of a quasi-variety is independent of the generating algebra}

\author{
BRIAN A. DAVEY AND RosS WILlARD
}

Abstract. We prove the claim made in the title of the paper.

It is to be expected that different generating algebras $\underline{\mathbf{D}}$ and $\underline{\mathbf{M}}$ for a quasi-variety will lead to different natural dualities. Indeed, this is the case-see the examples in [2]. But it would be most unfortunate if the very existence of a natural duality for a finitely generated quasivariety depended upon the choice of generator. Hence, the question below was posed in [2] and again in [4]. A positive solution to this question means that we may unambiguously refer to a finitely generated quasi-variety $\mathcal{A}$ as being dualisable provided $\mathcal{A}=\mathbb{I S P}(\underline{\mathbf{D}})$ for some finite dualisable algebra $\underline{\mathbf{D}}$.

- If $\underline{\mathbf{D}}$ and $\underline{\mathbf{M}}$ are finite algebras such that $\operatorname{ISP}(\underline{\mathbf{D}})=\operatorname{ISP}(\underline{\mathbf{M}})$, and if $\underline{\mathbf{D}}$ is dualisable, does it follow that $\underline{\mathbf{M}}$ is dualisable?

\section{THEOREM. Yes. ${ }^{1}$}

Proof. We refer the reader to Clark and Davey [1] for the necessary definitions and the basic theory of natural dualities. We recall only that $\underline{\mathbf{D}}$ is a finite algebra and $\underset{\sim}{\mathbf{D}}$ is an alter ego for $\underline{\mathbf{D}}$, that is, $\underset{\sim}{\mathbf{D}}$ is a topological structure whose universe is the universe of $\underline{\mathbf{D}}$, whose relations (if any) are non-empty subuniverses of the appropriate finite powers of $\underline{\mathbf{D}}$, whose operations and partial operations (if any) are such that their graphs are non-empty subuniverses of finite powers of $\underline{\mathbf{D}}$, and whose topology is discrete.

Let $\mathcal{A}=\mathbb{I S P}(\underline{\mathbf{D}})=\mathbb{I S P}(\underline{\mathbf{M}})$. Let $N=\left\{v_{0}, \ldots, v_{k-1}\right\}$ be a set of homomorphisms from $\underline{\mathbf{D}}$ to $\underline{\mathbf{M}}$ which separate the points of $D$. For example, an inefficient choice would be

Presented by Professor R. W. Quackenbush.

Received November 14, 1998; accepted in final form June 24, 1999.

1991 Mathematics Subject Classification: 08C15, 08C05, 18A40.

Key words and phrases: Natural duality, quasi-variety.

The second author gratefully acknowledges the support of the NSERC of Canada.

${ }^{1}$ This theorem was obtained independently, via a different proof, by M. J. Saramago [5]. 
$N=\mathcal{A}(\underline{\mathbf{D}}, \underline{\mathbf{M}})$. Let $v:=v_{0} \sqcap \cdots \sqcap v_{k-1}: \underline{\mathbf{D}} \rightarrow \underline{\mathbf{M}}^{k}$ be the natural product map and let $D_{v}:=v(D)$. Thus $D_{v}$ is a $k$-ary algebraic relation on $\underline{\mathbf{M}}$ and $v: \underline{\mathbf{D}} \cong \mathbf{D}_{v} \leqslant \underline{\mathbf{M}}^{k}$. Note that $v_{i}=\pi_{i} \circ v$ for $i<k$, where $\pi_{i}: \underline{\mathbf{M}}^{k} \rightarrow \underline{\mathbf{M}}$ is the $i$ th projection.

For $n \geqslant 1$ we construe $M^{k \times n}$ as the set of all matrices with $k$ rows and $n$ columns and with elements from $M$. If $\mathbf{A} \leqslant \underline{\mathbf{D}}^{n}$, then we define $v_{\mathbf{A}}^{n}: \mathbf{A} \rightarrow \underline{\mathbf{M}}^{k \times n}$ by $v_{\mathbf{A}}^{n}\left(a_{0}, \ldots, a_{n-1}\right)=$ $\left[v\left(a_{0}\right), \ldots, v\left(a_{n-1}\right)\right]$ where each $v\left(a_{i}\right) \in D_{v}$ is construed as a column vector, and we set $A_{v}=v_{\mathbf{A}}^{n}(A)$. Thus $v_{\mathbf{A}}^{n}: \mathbf{A} \cong \mathbf{A}_{v} \leqslant \underline{\mathbf{M}}^{k \times n}$, and $A_{v}$ is a $(k \times n)$-ary algebraic relation on $\underline{\mathbf{M}}$.

Similarly, choose homomorphisms $\omega_{0}, \ldots, \omega_{\ell-1}: \underline{\mathbf{M}} \rightarrow \underline{\mathbf{D}}$ which separate the points of $M$, let $\omega:=\omega_{0} \sqcap \cdots \sqcap \omega_{\ell-1}: \underline{\mathbf{M}} \rightarrow \underline{\mathbf{D}}^{\ell}$ be the induced embedding and define $M_{\omega}:=\omega(M)$ so that $\omega: \underline{\mathbf{M}} \cong \mathbf{M}_{\omega} \leqslant \underline{\mathbf{D}}^{\ell}$. Finally, define $\tau: \underline{\mathbf{M}} \cong\left(\mathbf{M}_{\omega}\right)_{v} \leqslant \underline{\mathbf{M}}^{k \times \ell}$ by $\tau=v_{\mathbf{M}_{\omega}}^{\ell} \circ \omega$. Thus $\sigma:=\tau^{-1}:\left(\mathbf{M}_{\omega}\right)_{v} \rightarrow \underline{\mathbf{M}}$ is a partial $(k \times \ell)$-ary algebraic operation on $\underline{\mathbf{M}}$.

We can now state our theorem more precisely.

Duality Transfer Theorem Assume that $\underline{\mathbf{D}}$ and $\underline{\mathbf{M}}$ are finite algebras which generate the same quasi-variety and let $\underset{\mathbf{D}}{\mathbf{D}}=\langle D ; R, \mathcal{T}\rangle$ be an alter ego which dualises $\underline{\mathbf{D}}$. Then $\underset{\sim}{\mathbf{M}}:=\left\langle M ; \sigma,\left\{r_{v} \mid r \in R\right\} \cup\left\{D_{v}\right\}, \mathcal{T}\right\rangle$ is an alter ego which dualises $\underline{\mathbf{M}}$.

Our assumption that $\underset{\sim}{\mathbf{D}}$ is relational, that is, that it has no operations or partial operations in its type, results in no loss of generality (see [1], Lemma 2.1.2). It should be clear that $\underset{\sim}{\mathbf{M}}$ is an alter ego for $\underline{\mathbf{M}}$ (modulo identification of $\underline{\mathbf{M}}^{k \times n}$ with $\underline{\mathbf{M}}^{k n}$ ). Given $\mathbf{A} \in \mathcal{A}$ we shall use $e_{\mathbf{A}}$ to denote the canonical embedding relative to the category $\mathcal{X}:=\mathbb{I S}_{\mathrm{c}} \mathbb{P}^{+}(\mathbf{D})$ and the functors $\mathcal{A}(-, \underline{\mathbf{D}})$ and $\mathcal{X}(-, \underline{\mathbf{D}})$, while we use $\mathrm{e}_{\mathbf{A}}^{\prime}$ for the canonical embedding relative to $\mathcal{X}^{\prime}:=\mathbb{I} \mathbb{S}_{\mathfrak{c}} \mathbb{P}^{+}(\underset{\sim}{\mathbf{M}})$ and the functors $\mathcal{A}(-, \underline{\mathbf{M}})$ and $\mathcal{X}^{\prime}(-, \underline{\mathbf{M}})$.

To prove that $\underset{\sim}{\mathbf{M}}$ dualises $\underline{\mathbf{M}}$, fix $\mathbf{A} \in \mathcal{A}$ and an $\mathcal{X}^{\prime}$-morphism $\alpha: \mathcal{A}(\mathbf{A}, \underline{\mathbf{M}}) \rightarrow \underline{\mathbf{M}}$. It must be shown that $\alpha=e_{\mathbf{A}}^{\prime}(a)$ for some $a \in A$. Note that if $x \in \mathcal{A}(\mathbf{A}, \underline{\mathbf{D}})$ then $v_{i} \circ x \in \mathcal{A}(\mathbf{A}, \underline{\mathbf{M}})$ for $i<k$, and $\left(\left(v_{0} \circ x\right)(a), \ldots,\left(v_{k-1} \circ x\right)(a)\right)=(v \circ x)(a) \in D_{v}$ for all $a \in A$. Since $D_{v}$ is in the type of $\underset{\sim}{\mathbf{M}}$ and $\alpha$ is an $\mathcal{X}^{\prime}$-morphism, it follows that $\left(\alpha\left(\nu_{0} \circ x\right), \ldots, \alpha\left(\nu_{k-1} \circ x\right)\right) \in D_{\nu}$. Thus we can define $\hat{\alpha}: \mathcal{A}(\mathbf{A}, \underline{\mathbf{D}}) \rightarrow D$ by $\hat{\alpha}(x)=v^{-1}\left(\alpha\left(v_{0} \circ x\right), \ldots, \alpha\left(v_{k-1} \circ x\right)\right)$. For each relation $r$ in the type of $\underset{\sim}{\mathbf{D}}$, it can be easily checked that $\hat{\alpha}$ preserves $r$, using the fact that $\alpha$ preserves $r_{\nu}$. Since $\alpha$ is continuous, there exists a finite subset $S \subseteq A$ such that if $y_{1}, y_{2} \in \mathcal{A}(\mathbf{A}, \underline{\mathbf{M}})$ and $y_{1} \uparrow_{s}=y_{2} \uparrow_{s}$, then $\alpha\left(y_{1}\right)=\alpha\left(y_{2}\right)$. It easily follows that if $x_{1}, x_{2} \in \mathcal{A}(\mathbf{A}, \underline{\mathbf{D}})$ and $x_{1} \uparrow_{s}=x_{2} \uparrow_{s}$, then $\hat{\alpha}\left(x_{1}\right)=\hat{\alpha}\left(x_{2}\right)$, which proves that $\hat{\alpha}$ is continuous. Hence $\hat{\alpha}$ is an $\mathcal{X}$-morphism from $\mathcal{A}(\mathbf{A}, \underline{\mathbf{D}})$ to $\underline{\mathbf{D}}$.

Since $\underset{\sim}{\mathbf{D}}$ dualises $\underline{\mathbf{D}}$, by assumption, there exists $a \in A$ such that $\hat{\alpha}=e_{\mathbf{A}}(a)$. We shall show that $\alpha=e_{\mathbf{A}}^{\prime}(a)$. First observe that if $x \in \mathcal{A}(\mathbf{A}, \underline{\mathbf{D}})$ and $i<k$, then

$$
\alpha\left(v_{i} \circ x\right)=\pi_{i}(v(\hat{\alpha}(x)))=v_{i}(\hat{\alpha}(x))=v_{i}(x(a))=e_{\mathbf{A}}^{\prime}\left(v_{i} \circ x\right) .
$$

Now let $y \in \mathcal{A}(\mathbf{A}, \underline{\mathbf{M}})$ be fixed, and consider the $k \times \ell$ matrix $\left[v_{i} \circ \omega_{j} \circ y\right]_{k \times \ell}$ of elements from $\mathcal{A}(\mathbf{A}, \underline{\mathbf{M}})$. Note that each entry in this matrix is of the form $v_{i} \circ x$ for some $x \in \mathcal{A}(\mathbf{A}, \underline{\mathbf{D}})$ and some $i<k$, so the previous observation applies to it. Secondly, note that for each $c \in A$ 


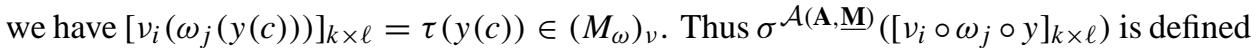
and equals $y$. Hence

$$
\begin{aligned}
\alpha(y) & =\alpha\left(\sigma^{\mathcal{A}(\mathbf{A}, \underline{\mathbf{M}})}\left(\left[v_{i} \circ \omega_{j} \circ y\right]_{k \times \ell}\right)\right) \\
& =\sigma\left(\left[\alpha\left(v_{i} \circ \omega_{j} \circ y\right)\right]_{k \times \ell}\right) \quad \text { as } \alpha \text { preserves } \sigma \\
& =\sigma\left(\left[v_{i}\left(\omega_{j}(y(a))\right)\right]_{k \times \ell}\right) \quad \text { by an earlier observation } \\
& =y(a),
\end{aligned}
$$

proving that $\alpha=e_{\mathbf{A}}^{\prime}(a)$.

When applying the Duality Transfer Theorem, we typically start with a 'minimal' generator of a quasi-variety $\mathcal{A}$, say $\underline{\mathbf{D}}$, and find an alter ego $\underset{\sim}{\mathbf{D}}$ for $\underline{\mathbf{D}}$ which yields a duality on $\mathcal{A}$. The Duality Transfer Theorem then provides an alter ego for any finite algebra $\underline{\mathbf{M}}$ which generates $\mathcal{A}$. An important special case occurs when we have an embedding $v: \underline{\mathbf{D}} \rightarrow \underline{\mathbf{M}}$ of the 'minimal' generator $\underline{\mathbf{D}}$ into $\underline{\mathbf{M}}$. (This occurs, for example, if $\underline{\mathbf{D}}$ is subdirectly irreducible and generates the same quasi-variety as $\underline{\mathbf{M}}$.) In this case, we have $k=1$ in the construction given above. To simplify the notation, we shall assume that $\underline{\mathbf{D}}$ is actually a subalgebra of $\underline{\mathbf{M}}$ so that $v$ is the inclusion map. Let $r$ be an algebraic relation on $\underline{\mathbf{D}}$. Since $\mathbf{r} \leqslant \underline{\mathbf{D}}^{n}$ and $\underline{\mathbf{D}} \leqslant \underline{\mathbf{M}}$, the relation $r_{v}$ is simply $r$ regarded as an algebraic relation on $\underline{\mathbf{M}}$. Following [2] (see also Section 7.7 of [1]), we shall denote the relation $r_{v}$ by $r_{D}$. The homomorphisms $\omega_{i}: \underline{\mathbf{M}} \rightarrow \underline{\mathbf{D}} \leqslant \underline{\mathbf{M}}$, which separate the points of $M$, may now be viewed as endomorphisms of $\underline{\mathbf{M}}$, the map $\omega:=\omega_{0} \sqcap \cdots \sqcap \omega_{\ell-1}: \underline{\mathbf{M}} \rightarrow \underline{\mathbf{D}}^{\ell} \leqslant \underline{\mathbf{M}}^{\ell}$ is an embedding of $\underline{\mathbf{M}}$ into $\underline{\mathbf{M}}^{\ell}$ and $\sigma:=\omega^{-1}: \omega(\underline{\mathbf{M}}) \rightarrow \underline{\mathbf{M}}$ is an $\ell$-ary algebraic partial operation on $\underline{\mathbf{M}}$. Thus, we have the following corollary of the Duality Transfer Theorem.

Subalgebra Duality Transfer Theorem Let $\underline{\mathbf{D}}$ and $\underline{\mathbf{M}}$ be finite and assume that $\underline{\mathbf{D}}$ is a subalgebra of $\underline{\mathbf{M}}$ and that $\underline{\mathbf{M}} \in \mathbb{I S P}(\underline{\mathbf{D}})$. If $\underline{\mathbf{D}}=\langle D ; R, \mathcal{T}\rangle$ is an alter ego which dualises $\underline{\mathbf{D}}$, then $\mathbf{M}:=\left\langle M ; \sigma,\left\{r_{D} \mid r \in R\right\} \cup\{D\}, \mathcal{T}\right\rangle$ is an alter ego which dualises $\mathbf{M}$.

It is interesting to compare this with the corresponding result in Saramago [5] which states that, under the same assumptions on $\underline{\mathbf{D}}$ and $\underline{\mathbf{M}}$, a dualising alter ego for $\underline{\mathbf{M}}$ is given by $\underset{\sim}{\mathbf{M}^{\prime}}:=\left\langle M ;\left\{\omega_{j} \mid j<\ell\right\},\left\{r_{D} \mid r \in R\right\} \cup\{D\}, \mathcal{T}\right\rangle$.

Recently, Davey and Haviar [3] have shown that the algebraic partial operation $\sigma$ : $\omega(\underline{\mathbf{M}}) \rightarrow \underline{\mathbf{M}}$ plays a vital role in the transfer of a strong duality from $\underline{\mathbf{D}}$ to $\underline{\mathbf{M}}$. They prove that, under the assumptions of the Subalgebra Duality Transfer Theorem, an alter ego which strongly dualises $\underline{\mathbf{D}}$ may be lifted to an alter ego which strongly dualises $\underline{\mathbf{M}}$ by simply adding the endomorphisms $\omega_{0}, \ldots, \omega_{\ell-1}$ along with partial operation $\sigma$.

\section{REFERENCES}

[1] Clark, D. M. and Davey, B. A., Natural Dualities for the Working Algebraist, Cambridge University Press, Cambridge, 1998. 
[2] DAVEY, B. A., Dualisability in general and endodualisability in particular, Logic and algebra (Pontignano, 1994), (A. Ursini and P. Aglianò, eds), Lecture Notes in Pure and Appl. Math. 180, Dekker, New York, 1996, 437-455.

[3] DAVEY, B. A. and HAVIAR, M., A schizophrenic operation which aids the efficient transfer of strong dualities, submitted.

[4] DAvey, B. A., IDZIAK, P. M., LAMPe, W. A. and McNulty, G. F., Dualizability and graph algebras, Discrete Math., 214 (2000), 145-172.

[5] Saramago, M. J., Some remarks on dualisability and endodualisability, Algebra Univers. 43 (2000), 197-212.

\author{
School of Mathematics \\ La Trobe University \\ Victoria 3086 \\ Australia \\ e-mail: B.Davey@latrobe.edu.au \\ Department of Pure Mathematics \\ University of Waterloo \\ Waterloo \\ Ontario N2L $3 G 1$ \\ Canada \\ e-mail:rdwillar@gillian.math.uwaterloo.ca
}

To access this journal online:

(2P) http://www.birkhauser.ch 